International Journal for Parasitology, 1995, v.25, n.10, pp.1179-1184.

ISSN: 0020-7519

DOI: $10.1016 / 0020-7519(95) 00051-3$

http://www.elsevier.com/wps/find/journaldescription.cws home/353/description\#description

(C) 1995 Australian Society for Parasitology Elsevier Science Ltd

\title{
Increased Coprophagic Activity of the Beetle, Tenebrio molitor, on Feces Containing Eggs of the Tapeworm, Hymenolepis diminuta
}

\author{
Peter W. Pappas, Elizabeth A. Marschall, Sarah E. Morrison, Geraldine M. \\ Durka and Cary S. Daniel \\ Department of Zoology, The Ohio State University.
}

\begin{abstract}
.
When provided with fecal pellets from uninfected (control) rats and rats infected with the tapeworm Hymenolepis diminuta, more fed and starved (72 h) female and starved male Tenebrio molitor fed on fecal pellets from infected- than from control rats; compared to fecal pellets from controls rats, fed males avoided the infective fecal pellets. Uninfective and infective fecal pellets had similar moisture contents, so increased coprophagic activity was not due to differences in moisture content. Fed and starved males and females were fed on fecal pellets containing tapeworm eggs and examined for cysticercoids. Significantly greater numbers of starved beetles than fed beetles were infected with cysticercoids, but the numbers of infected males and females within each treatment were not significantly different. On the other hand, males contained significantly greater numbers of cysticercoids than did females, and there was no significant difference between the numbers of cysticercoids recovered from fed and starved beetles. The data support the hypothesis that the feeding behavior of T. molitor on rat feces is altered by the presence of tapeworm eggs. The data demonstrated further that transmission dynamics are affected by a complex interaction of the beetle's sex and nutritional status.
\end{abstract}

\section{INTRODUCTION}

The successful completion of the life-cycles of many parasitic organisms requires that successive hosts be infected by ingesting some type of infective stage, such as an "egg" passed in the feces of a previous host or a larval (immature) stage present in an intermediate host. Any adaptation on the part of a parasite that increases the probability that these infective stages will be ingested will, therefore, favor completion of the parasite's life-cycle.

In those instances in which an infective stage is a larva found within a living host, adaptations favoring the transmission of a larva could include the parasite causing changes in a host's appearance, physiology or behavior, so that the host is more susceptible to predation (reviewed by Toft, Aeschlimann \& Bolis, 1991). Examples of such adaptations are found in the trematodes, Dicrocoelium dendriticum (in which the intermediate host's behavior is affected) and Leucochloridium macrostoma (in which the intermediate host's appearance is altered). In those instances in which an infective stage consists of an egg (containing a larva) that is passed in the host's feces, and in which transmission depends on the egg being ingested by an appropriate intermediate host, possible adaptations that increase the probability of transmission are less 
obvious. One possible adaptation is for a parasite to produce prodigious numbers of eggs; this appears to be a common strategy among some groups of parasites, as virtually all adult helminth parasites contain little more than reproductive organs and eggs. Another possible adaptation would be for the adult parasite to produce a chemical that is passed in the host's feces that would attract the intermediate host to feces contaminated with eggs or make the feces more palatable to the host. Support for this hypothesis was provided by Evans et al. (1992) when they demonstrated that the beetle, Tribolium confusum, an intermediate host for the tapeworm, Hymenolepis diminuta, preferentially ingests rodent feces contaminated with the tapeworm's eggs. However, the experimental design used by Evans et al. (1992) was limited in several aspects; they used beetles of unknown age and sex (their beetles were collected "randomly" from cultures); their foraging experiments were not performed under conditions of constant lighting (the experiments were run in "reduced light", but every 20 min the light intensity was increased to permit counting of the beetles); the foraging experiments were only replicated 4 times; and data were only collected at 20 min intervals over a $4 \mathrm{~h}$ period. The purpose of the present investigation was to examine in greater detail the hypothesis that tapeworms can modify the feeding behavior of their intermediate host and, thereby, affect transmission dynamics. For this study, the coprophagic behavior of the beetle Tenebrio molitor, a normal intermediate host for $H$. diminuta, was examined. These experiments used beetles of known sex and age, and were designed to be run under conditions of constant illumination (red light conditions). Moreover, the beetles' movements were monitored continuously so that temporal changes in the beetles' behavior would be easier to detect, and most experiments were replicated 10 times to provide larger data sets for more robust statistical analyses.

\section{MATERIALS AND METHODS}

Maintenance of animals and collection of feces. The "OSU Strain" (Pappas \& Leiby, 1986) of $H$. diminuta was maintained in male Sprague-Dawley rats and beetles (T. molitor). Beetles were maintained at $26^{\circ} \mathrm{C}$ on wheat bran supplemented with Brewer's Yeast, and small pieces of potato were added to the cultures on a regular basis. Cultures were maintained on a 4:20 h light: dark cycle. Pupae were removed from the cultures, and the male and female pupae (Bhattacharya, Ameel \& Waldbauer, 1970) were placed in separate dishes containing wheat bran, yeast, and potato. The beetles that emerged during a $48 \mathrm{~h}$ period were collected, separated into groups (10 beetles/group), and maintained as above until they were 14-18 days old. Some experiments used beetles that had been starved for $72 \mathrm{~h}$ before placing them in the test arena (see Preference Experiments, below). To obtain feces containing eggs of $H$. diminuta, six male Sprague-Dawley rats weighing 80-100 g were each infected with 30 cysticercoids and maintained on commercial rodent chow and water ad libitum. Three additional rats, obtained from the same commercial source and of identical age, were maintained under conditions identical to those of the infected rats to serve as a source of uninfective (control) feces; feces from infected rats were not used prior to 20 days post-infection (p.i.). To obtain feces, cages containing infected or uninfected rats were suspended over separate, clean plastic trays, and the fecal pellets that were passed by the rats during 15 min periods between 0630 and $0730 \mathrm{~h}$ were collected; this was done to prevent contamination of the feces with urine, to minimize dehydration, and to minimize temporal variations in the numbers of eggs in, and chemical composition of, the feces. Single fecal pellets were used as bait, and feces that were not used 
immediately were discarded. After each experiment, the fecal pellets from the infected rats were examined microscopically to verify the presence of $H$. diminuta eggs.

Preference experiments. To determine the effect of sex and feeding history (fed versus starved) on the beetles' preferences for feeding on uninfective and infective fecal pellets, a $2 \times 2$ factorial design of preference experiment was used. (The beetles' behavior was characterized as "preference for" as opposed to "attraction to", since there are no data to indicate that beetles are actually attracted to fecal pellets or simply encounter them randomly.) Each of the 4 combinations of sex (males versus females) and feeding history (fed versus starved) was replicated 10 times. For each experiment a group of 10 beetles was removed from the incubator (under red light) at the end of the dark cycle and placed in an enclosure in the middle of a test arena (Fig. 1). Beetles were provided with two alternative baits (e.g. feces with and without tapeworm eggs); one type of bait (a single fecal pellet) was placed in areas 1 and 3, and the alternate bait was placed in areas 2 and 4 . After 15 min the enclosure was removed, and the beetles' movements were recorded continuously for 60 min under red illumination; each group of beetles was discarded after a single experiment. The recordings were played back, and the total number of beetles on the alternate baits (i.e. baits $1+3$ and baits $2+4$ ) at 1 min intervals were recorded. The total number of observations of beetles at each type of bait was summed over the entire 60 min experiment. To determine if the beetles exhibited temporal changes over the 60 min observation period, the data for (1) the first $10 \mathrm{~min}$ and (2) successive $10 \mathrm{~min}$ intervals (1-10 min, 11-20 min, etc.) were also analysed. Experiments $(n=10)$ without any food (bait) present in the experimental arena served as a control for non-feeding related preference. Split-plot analyses of variance (ANOVAs) were used to test for differences between the numbers of beetles at the infective and uninfective baits.

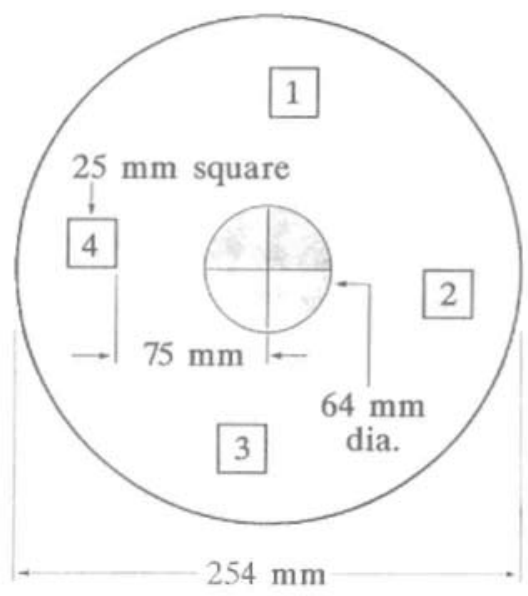

Fig. 1. Diagrammatic representation of the test arena. In a typical experiment, the beetles were provided with alternative baits placed in adjacent bait areas (e.g. infective feces in 1 and 3, and uninfective feces in 2 and 4). 
Analysis of moisture content and rehydration of fecal pellets. Casual observations of the behavior of $T$. molitor indicated that the beetles preferred moist food sources. This suggested that the basis of the beetles' preference for or avoidance of feces from infected rats might be due to differences in the feces' moisture content. To test this hypothesis, separate wire-bottom cages containing 3 infected or 3 control rats were suspended over clean, dry plastic pans, and the fecal pellets that were passed were collected immediately, weighed, vacuum-dried at room temperature for $24 \mathrm{~h}$, and weighed again. Fecal pellets were only collected between 0630 and 0730 h, and fecal pellets were collected on a daily basis (using the same control and infected rats) until 50 fecal pellets from infected and control rats were collected. Fecal pellets were rehydrated by placing them in individual plastic Petri dishes, the bottoms of which were lined with filter paper saturated with distilled water; the Petri dishes were covered and stored at $4^{\circ} \mathrm{C}$. The saturated filter paper was replaced at $12 \mathrm{~h}$ intervals, and the amount of water reabsorbed by the fecal pellets was determined by weighing the pellets at $24 \mathrm{~h}$ intervals.

The potential effects of dehydration and rehydration of fecal pellets on the beetles' preferences were determined by completing three additional sets of preference experiments ( $n=10$ for each set) in which the baits consisted of (1) fresh versus rehydrated uninfective fecal pellets, (2) fresh versus rehydrated infective fecal pellets, and (3) rehydrated uninfective versus rehydrated infective fecal pellets. The rehydrated fecal pellets used in these experiments were vacuum-dried and then rehydrated for 24 h.

Consequences of preference: amount of feces ingested. To quantify the amount of fecal material actually ingested by beetles, preference experiments $(n=10)$ were run as described above, but the individual fecal pellets were weighed before and after the experiments. However, even when not fed upon by beetles, fecal pellets decreased in weight during a 60 min period due to dehydration. Thus, to correct for the weight loss due to dehydration, 40 fecal pellets from infected and uninfected rats were collected and weighed, and weighed again after $60 \mathrm{~min}$. The weights of the fecal pellets on which beetles fed were corrected for weight loss due to dehydration using these data. For each replicate of each sex and feeding history combination, the difference between the amount ingested from infective and uninfective pellets was calculated, and the differences were compared using a $t$-test. In addition, split-plot ANOVAs were used to test for the effects of sex, feeding history, and whether feces contained eggs, on the amount of fecal material ingested.

Prevalence and intensity of infection. The prevalence and intensity of cysticercoid infections in beetles that had fed on infective feces, and the effects of the beetles' sex and feeding history, were examined. For these experiments $(n=10), 5$ male and 5 female beetles were marked with small dots of water-soluble paint for later identification and then allowed to feed simultaneously for $1 \mathrm{~h}$ on a single fecal pellet containing $H$. diminuta eggs. Since individual fecal pellets might contain different numbers of eggs, a single fecal pellet was used as bait in each experiment so that the male and female beetles in a single experiment were exposed to the same number of eggs; all fecal pellets used in these experiments were collected at the same time of day to minimize temporal variations in the number of eggs/fecal pellet. (In the absence of empirical data indicating otherwise, it was assumed that the eggs within the fecal pellets were distributed randomly.) Immediately after feeding, the male and female beetles were placed in separate containers containing wheat bran. Not earlier than 14 days after feeding, the number of cysticercoids in each beetle was determined. 
Differences in prevalence and intensity of infection were compared by using (1) a $x^{2}$ test to compare frequencies of infected individuals among different sex and feeding history combinations, and (2) paired $t$-tests to compare mean numbers of cysticercoids per individual between males and females. For the $x^{2}$-test, only frequencies of individuals with and without cysticercoids were compared. For the $t$-tests, the means for each sex in each experiment were calculated and paired for each replicate and then compared.

\section{RESULTS}

Initial control experiments were conducted without bait in the arena. These demonstrated that beetles showed no preference for those areas in which uninfective or infective baits were placed, and that the number of beetles associated with the bait areas was independent of sex and feeding history (controls of Fig. 2). In all experiments in which feces were used as bait and the results of the entire $60 \mathrm{~min}$ observation period were compared, the number of beetles associated with the bait was significantly greater than in the control experiments, but male and female beetles reacted quite differently to uninfective and infective feces, and their behaviors were also different depending on whether or not they had been starved (Fig. 2; $P<0.05$ for each of the differences within groups). Similar results were obtained when the first 10 min of the observation period were compared with the entire 60 min observation period, or when successive 10 min intervals were compared (data not shown).

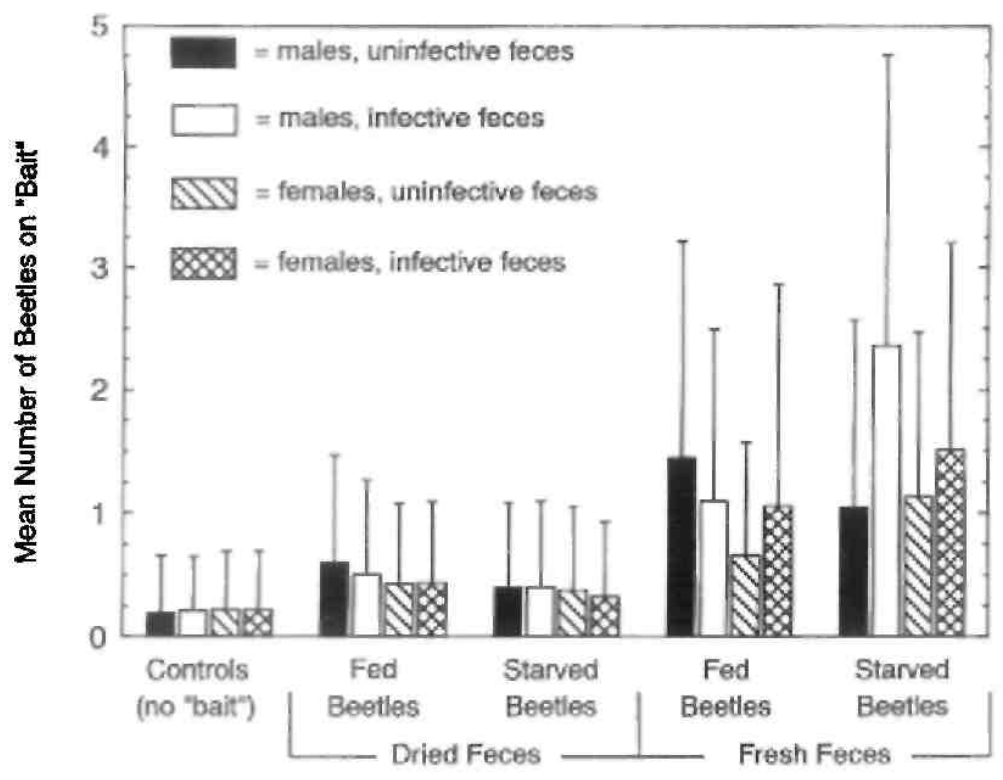

Fig. 2. Mean ( \pm S.D.) number of occurrences of beetles at each type of bait (or bait area for control experiments). Each bar is the mean of the sum of 60 observations ( 1 per min) from 10 replicate experiments. 


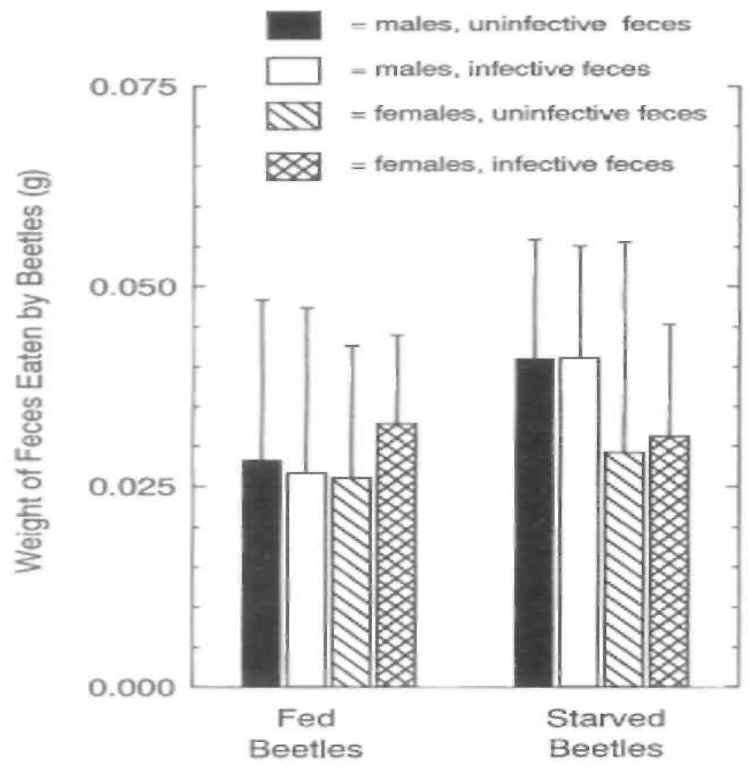

Fig. 3. Summary of data for the ingestion of fecal material by beetles. Each bar represents the mean ( \pm S.D.) of 20 fecal pellets (10 experiments, each containing 2 fecal pellets).

The amounts of uninfective and infective fecal material eaten by beetles were not significantly different $(P>0.1$ for all comparisons of means within experiments; Fig. 3). Also, there were no significant effects of sex, feeding history, or the presence of eggs on the amount of fecal material ingested, but the same trends seen in Fig. 2 were noted in these data (Fig. 3). Losses in weight due to dehydration of uninfective and infective fecal pellets during a $60 \mathrm{~min}$ period amounted to $11.7 \%$ (S.D. $=4.4, N=$ 40 ) and $10.8 \%$ (S.D. $=4.2, N=40$ ) of the initial wet weights ( $t$-tests, $P>0.3$ ). The weights of fecal pellets ranged between 200 and $600 \mathrm{mg}$, so dehydration alone resulted in decreases in wet weight of $20-60 \mathrm{mg}$, and these values accounted for about $50 \%$ of the total decrease in weight wet during the $60 \mathrm{~min}$ experiments. These large decreases in wet weight due to dehydration may have obscured subtle differences between the data for males and females or fed and starved beetles; thus, the data presented in Fig. 3 must be interpreted with some caution. 

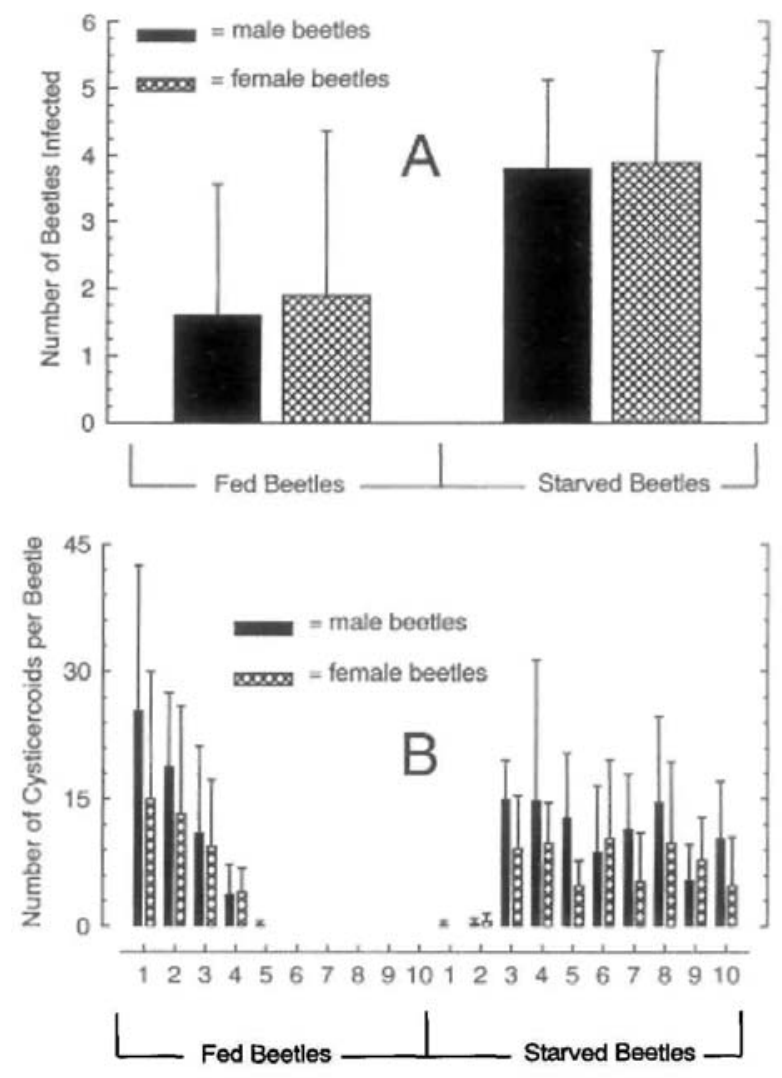

Fig. 4. Recovery of cysticercoids from beetles that had fed on feces containing tapeworm eggs. Graph A (top) shows the mean ( \pm S.D.) number of beetles infected with cysticercoids $(n=10,5$ males and 5 females in each experiment). Graph B (bottom) shows the mean ( \pm S.D.) number of cysticercoids recovered from males and females in individual experiments. (The experiments were not done in the order in which they are listed [1-10], and no temporal relationship should be inferred from these data.)

Significant differences were noted between male and female and fed and starved beetles when beetles were allowed to feed on infective fecal pellets and later examined for cysticercoids (Fig. 4). When the numbers of uninfected and infected beetles in each group were analysed (i.e. the prevalence of infection), and the numbers of cysticercoids recovered were disregarded, males and females within treatments (fed versus starved) did not differ (for fed and starved beetles, $P>0.4$ and $P>0.9$, respectively, using $x^{2}$ - tests), but significantly more starved than fed beetles were infected (for both sexes, $P<0.001$ using $x^{2}$-tests). However, when the numbers of cysticercoids recovered from the beetles were analysed (i.e. intensity of infection), fed and starved beetles did not differ significantly (due primarily to the high variability among the experiments using fed beetles), but males contained significantly greater numbers of cysticercoids than did females ( $t$-test, $P<0.01)$. 


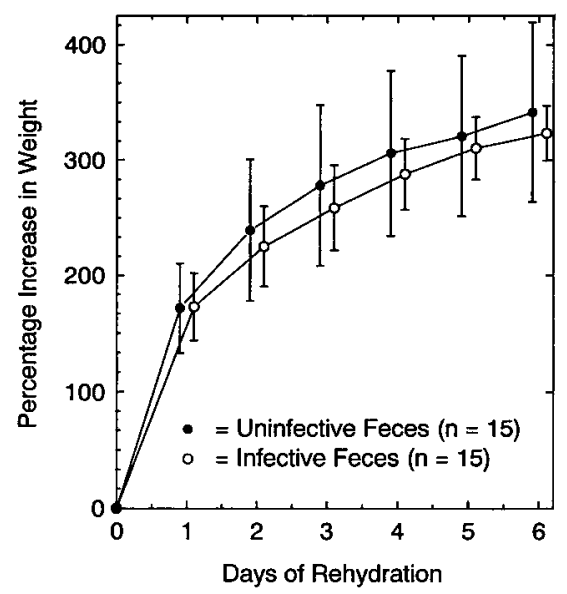

Fig. 5. Rehydration of vacuum-dried fecal pellets without (uninfective) and with (infective) eggs of Hymenolepis diminuta. The amount of water reabsorbed (mean \pm S.D.) by the pellets was calculated as a percentage of the pellets' dried weights.

When provided with a choice of dried and moist (fresh) feces, beetles preferred the latter (Fig. 2). This suggested that beetles might prefer feces from infected rats simply because of a higher moisture content. However, the moisture contents of feces from uninfected and infected rats were determined to be $52.1 \%$ (S.D. $=4.7, n=50)$ and $51.1 \%$ (S.D. $=4.2, n=50$ ), respectively, and the difference in moisture content was not statistically significant ( $t$-test, $P=0.27$ ).

Under natural conditions, fecal pellets could dry out completely and then be rehydrated, and this could result in changes in the composition of the fecal pellets that might affect the beetles' preferences. Thus, the amounts of water reabsorbed by vacuum-dried uninfective and infective fecal pellets were determined. On the basis of their dry weights, vacuum-dried uninfective and infective fecal pellets absorbed virtually identical amounts of water (Fig. $5 ; n=15$ uninfective and infective fecal pellets; $P>0.1$ for all comparisons). When beetles were provided with a choice of fresh or rehydrated uninfective feces, or fresh or rehydrated infective feces, they always preferred the rehydrated feces (split-plot ANOVAs, $P<0.05$ in all cases) (Fig. $6)$. 


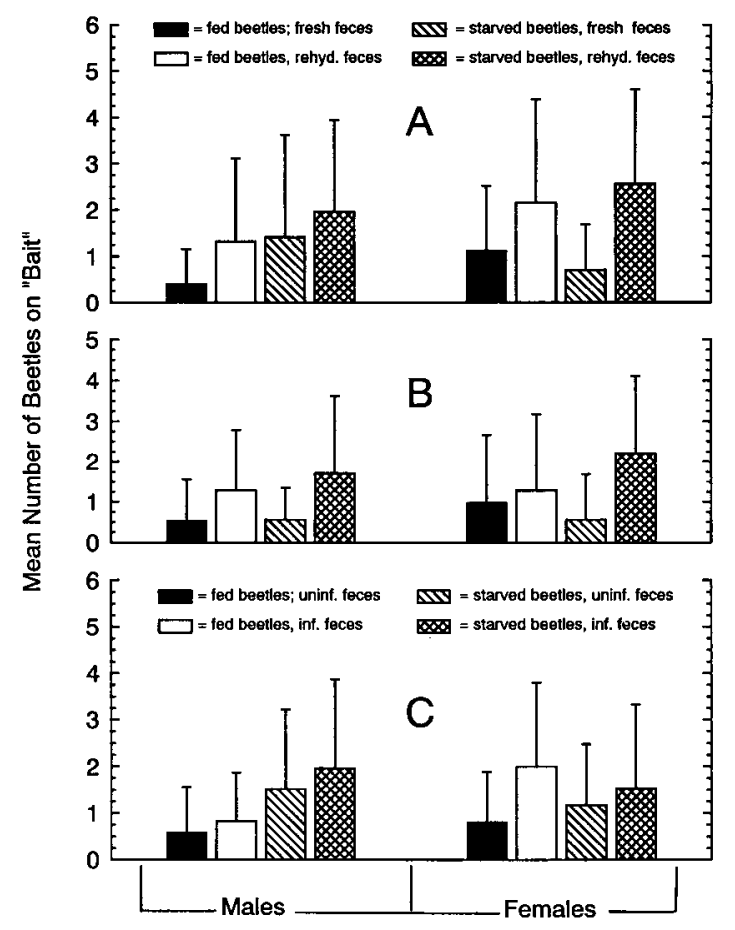

Fig. 6. Mean ( \pm S.D.) number of occurrences of beetles at each type of bait for experiments comparing the preferences of male and female beetles. Graphs A and B compare preferences for uninfective and infective feces, respectively (same symbols used in both graphs). Graph C compares the preferences of male and female beetles for rehydrated uninfective and infective pellets. Each bar is the mean of the sum of 60 observations (1 per min) from 10 replicate experiments.

The basis for this preference remains unknown. More important was the observation that beetles, when provided with a choice of rehydrated uninfective and infective fecal pellets, preferred the rehydrated infective fecal pellets (split-plot ANOVAs, $P<0.05$ in all cases). These data supported the data presented in Fig. 2 (i.e. beetles preferred infective fecal pellets) and the observation that the water content of the fecal pellets was not the basis for this preference (rehydrated uninfective and infective pellets reabsorbed similar amounts of water and, therefore, had similar moisture contents; Fig. 5).

\section{DISCUSSION}

The data of the current study demonstrate clearly that beetles, when provided with feces without and with $H$. diminuta eggs, prefer feces containing the tapeworm's eggs. These data suggest, therefore, that the feces from uninfected and infected rats are different, and that beetles can distinguish this difference, but the basis of the beetles' ability to differentiate uninfective versus infective feces is not the moisture content of the feces. An alternative hypothesis, as proposed by Evans et al. (1992), is that the feces from infected rats contain an attractant that is not present in the feces of uninfected rats. This attractant might be produced directly by the tapeworm (e.g. an excretory or secretory product), or it might be produced by the host as a result of the tapeworm altering the host's gastrointestinal physiology. The nature of this attractant remains unknown. However, the fact that beetles prefer infective feces compared to uninfective feces even when the feces have been vacuum-dried and rehydrated suggests 
that the attractant, if present, is not volatile. Despite the fact that the basis of the beetles' preference for infective fecal pellets remains unclear, the data of the current study support the hypothesis of Evans et al. (1992) "that a cestode can direct the feeding behavior of its potential intermediate host in a manner that benefits its transmission."

Evans et al. (1992) also demonstrated that the age and sex of an intermediate host ( $T$. confusum in their study) might play an important role in its ability to serve as an intermediate host, and such differences also exist in T. molitor (see also Hurd \& Arme, 1987). However, it is unclear at this time whether males are actually more "susceptible" to infection than are females (that is, if cysticercoids have a higher rate of survival in males than females), or whether this observation reflects differences in the feeding behavior of the sexes. The egg of $H$. diminuta is covered by a highly resistant "shell". The shell must be mechanically disrupted by the beetle's mouthparts as the egg is eaten or the larva (oncosphere) within will not be activated, and the beetle cannot be infected (reviewed by Lethbridge, 1980). Thus, while the differences in prevalences and infection intensities noted between males and females, and beetles of different ages, might reflect physiological differences in the beetles, they might also be explained by behavioral differences that control how well a beetle "chews its food".

Transmission of $H$. diminuta between the definitive and intermediate hosts seemingly involves a number of complex interactions ranging from the production of an attractant that is present in the definitive host's feces as well as the sex and nutritional status (and probably age) of the intermediate host. Additional studies are required to determine how these interrelated characteristics affect the overall transmission dynamics of this tapeworm species.

\section{References}

Bhattacharya A. K., Ameel J. J. \& Waldbauer G. P. 1970. A method for sexing living pupal and adult yellow mealworms. Annals of the Entomological Society of America 63: 1783.

Evans W. S., Hardy M. C, Singh R., Moodie G. E. \& Cote J. J. 1992. Effect of the rat tapeworm, Hymenolepis diminuta, on the coprophagic activity of its intermediate host, Tribolium confusum. Canadian Journal of Zoology 70:2311-2314.

Hurd H. \& Arme C. 1987. Hymenolepis diminuta (Cestoda): the role of intermediate host sex in the establishment, growth and development of metacestodes in Tenebrio molitor (Coleoptera). Helminthologia 24: 23-31.

Lethbridge R. C. 1980. The biology of the oncosphere of cyclophyllidean cestodes. Helminthological Abstracts 49A: 59-72.

Pappas P. W. \& Leiby D. A. 1986. Variation in the sizes of eggs and oncospheres and the numbers and distributions of testes in the tapeworm, Hymenolepis diminuta. Journal of Parasitology 72: 383391.

Toft C. A., Aeschlimann A. \& Bolis L. (Eds) 1991. Parasite-Host Associations: Coexistence of Conflict? Oxford University Press, Oxford. 